\title{
In Tube Ultrasonic and Air Assisted Liquid-Liquid Microextraction-Gas Chromatography-Mass Spectrometry Determination: a Novel Method for the Determination of Phthalate Esters in Aqueous Samples
}

\author{
Abolfazl Farahani, ${ }^{a}$ Majid Ramezani, ${ }^{*, a}$ Jalal Hassan $^{b}$ and Ali Niazi $^{a}$ \\ ${ }^{a}$ Department of Chemistry, Arak Branch, Islamic Azad University, Arak, Iran \\ ${ }^{b}$ Division of Toxicology, Department of Basic Sciences, Faculty of Veterinary Medicine, \\ University of Tehran, Tehran, Iran
}

\begin{abstract}
In this study, a highly sensitive and reliable microextraction method, known as in tube ultrasonic and air assisted liquid-liquid microextraction (IT-UAA-LLME), was developed for the preconcentration of some phthalate esters, such as dimethyl phthalate, diethyl phthalate (DEP), di-butyl-phthalate (DBP), bis(2-ethylhexyl) phthalate (BEHP), butyl benzyl phthalate (BBP), and di $n$-octyl-phthalate (DNOP), prior to their determination using gas chromatography-mass spectrometry (GC-MS). In this technique, the extraction solvent was dispersed into the aqueous sample solution using an ultrasonic bath. The effects of solvent type, aeration time and salt concentration were investigated. After extraction, an organic solvent was injected into the GC-MS instrument for quantification. Under optimum conditions, the enrichment factors ranged from 100 to 330 . The calibration curves showed good linearity $\left(r^{2}>0.99\right)$ and limit of detections were obtained in the range of $0.1-2.1 \mathrm{ng} \mathrm{mL}^{-1}$ in the working concentration ranges. The method was successfully applied for determination of six phthalate esters in real samples with complex matrices with relative standard deviation less than $8 \%$, recovery $88-112 \%$ and thus, satisfactory results were obtained.
\end{abstract}

Keywords: gas chromatography-mass spectrometry, in tube ultrasonic and air assisted liquidliquid microextraction, phthalate esters, water samples

\section{Introduction}

Phthalates or phthalate esters (PEs) are esters of phthalic acid and are mainly used as plasticizers (substances added to plastics to increase their flexibility, transparency, durability, and longevity). This material can gain entrance into the human body in many ways since huge amounts are usually found in packaged food. Continuous intake of this material may result in the incidence of cancer, including breast cancer in women and prostate cancer in men, as well as other symptoms. ${ }^{1}$ The ester of 1,2-benzenedicarboxylic acid is the most common phthalate. As shown in Figure 1, $\mathrm{R}$ and $\mathrm{R}$ ' represent alkyl chains that may or may not be the same and may contain oxygen. Potentially an infinite number of isomers are possible.

PEs with high molecular weight, such as bis(2-ethylhexyl) phthalate (BEHP), are primarily used as plasticizers to soften PVC products, while PEs with low molecular weight, such as diethyl phthalate (DEP), di-butyl-phthalate (DBP)

*e-mail: m-ramezani@iau-arak.ac.ir<smiles>[R]OC(=O)c1ccccc1C(=O)O[R]</smiles>

Figure 1. General chemical structure of phthalates ( $R$ and $R$ ' are general placeholders).

and butyl benzyl phthalate (BBP), are widely used as solvents to hold color and scent in various consumable and personal care products.

Several agencies, such as the US Environmental Protection Agency (EPA), classified PEs as priority pollutants. ${ }^{2}$ Therefore, a reliable and highly sensitive method for the identification and quantification of PEs from food, environmental water and other matrices is particularly important. BEHP is a high production volume chemical. The US EPA limits for BEHP in drinking water is $6 \mu \mathrm{g} \mathrm{L}^{-1}$. 
According to the World Health Organization (WHO), ${ }^{3}$ a guideline has been established to consider the limit value of $8.0 \mu \mathrm{g} \mathrm{L}{ }^{-1}$ phthalate esters in fresh and drinking waters. Gas chromatography (GC) and liquid chromatography (LC) coupled with various detectors were frequently employed for the determination of PEs. Most PEs are semi-volatile, thermo stable and non-polar compounds. Therefore, GC with a capillary column is commonly used as a separation platform for PEs and mass spectrometry (MS) has now become the routine detection technique for PEs. Most samples are not suitable for direct introduction into analytical instruments. For this reason, the sample preparation procedure is an important step in an analytical study. Up to now, various pretreatment techniques have been carried out to extract PEs from different samples, ${ }^{4}$ such as ionic liquid cold-induced aggregation dispersive liquidliquid microextraction (IL-CIA-DLLME), ${ }^{5}$ dispersive liquid-liquid microextraction (DLLME), ${ }^{6}$ graphenebased magnetic nanocomposite, ${ }^{7}$ and magnetic carbon nanotubes (CNTs) ${ }^{8}$ Recently, an air-assisted liquid-liquid microextraction method (AALLME) as a new version of DLLME was developed for extraction and preconcentration of some compounds in aqueous samples. ${ }^{9-11}$ In this method, a solvent-coated bubble is used to contact organic and aqueous phases. Aeration causes the extraction solvent to form a thin layer on bubbles and thus results in an increase in the contact area between the two phases. Due to the increasing interfacial area between the extraction solvent and aqueous phase, analytes can be extracted into the organic phase within a short time with higher efficiency. In another study, vortex and air bubbling were used to improve the microextraction method. ${ }^{12}$

Ultrasonic radiation is a powerful aid, which can accelerate various steps of the analytical process, and thus, ultrasound-assisted emulsification-microextraction (USAEME) has been used as an alternative method for conventional liquid-liquid extraction (LLE). ${ }^{13-16}$ Accordingly, the production of tiny air bubble and good dispersion of extraction solvent in the aqueous phase are important.

Air-assisted liquid-liquid microextraction (AALLME) was used for first time for extraction of some phthalate esters, which used a new version of DLLME method for extraction and preconcentration of phthalate esters, dimethyl phthalate (DMP), DEP, di-isobutyl phthalate (DIBP), di- $n$-butyl phthalate (DNBP), and bis(2-ethylhexyl) phthalate (BEHP), from aqueous samples prior to gas chromatography-flame ionization detection (GC-FID). ${ }^{17}$

In this study, a novel technique called the in tube ultrasonic and air assisted liquid-liquid microextraction (IT-UAA-LLME) was developed for the determination of trace levels of six phthalate esters in water and cola samples. Unlike the DLLME method, extraction was performed without the use of disperser solvent and centrifugation step. After mixing of sample solution and extraction solvent using ultrasonic bath, the cloudy mixture was transferred to a long tube and subjected to aeration process with tiny air bubble. Aeration leads to phase separation and increases analytes transfer to organic phase. Finally, the upper organic phase was removed and injected into GC-MS system. The influences of the different experimental parameters on the extraction efficiency of the model analytes were studied and optimized.

\section{Experimental}

\section{Apparatus and chemicals}

All PEs (DMP, DEP, DBP, BEHP, BBP, and di $n$-octylphthalate (DNOP)) were purchased from Sigma-Aldrich (St. Louis, USA). Four mineral waters and one Cola (carbonated drink) sample were purchased randomly from supermarkets in Tehran and stored at $4{ }^{\circ} \mathrm{C}$ before sample preparation. The cola sample was degassed in ultrasonic bath for $5.0 \mathrm{~min}$. To avoid contamination, all glassware used was washed using ethanol. An ultrasonic bath from Kodous company, China, was also used. The instrument used for GC-MS analysis was an Agilent (Agilent Technologies, CA, USA) 7890 gas chromatograph equipped with a 5975 mass selective detector quadruple mass spectrometer. The gas chromatograph was fitted with a DB-5 ms capillary column ( $30 \mathrm{~m}, 0.25 \mathrm{~mm}$ i.d., $0.25 \mathrm{~mm}$ film thickness).

\section{Chromatographic procedure}

The GC-MS temperatures were as following: injector temperature $290{ }^{\circ} \mathrm{C}$; initial oven temperature $70{ }^{\circ} \mathrm{C}$, held for $1 \mathrm{~min}$, increased to $300{ }^{\circ} \mathrm{C}$ at the rate of $10{ }^{\circ} \mathrm{C} \mathrm{min}^{-1}$, and held for $7 \mathrm{~min}$. The inlet was operated in splitless mode. The temperature of the transfer line was maintained at $300{ }^{\circ} \mathrm{C}$. Helium $(99.9999 \%)$ was used as carrier gas at

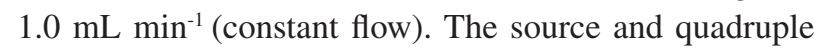
temperatures were kept at 230 and $150{ }^{\circ} \mathrm{C}$, respectively. Their electronic beam energy, using spectrometer, was set at $70 \mathrm{eV}$. The compounds were quantified using selected ion monitoring (SIM) mode. One quantitation and two qualifier ions were monitored for each compound (Table 1).

\section{General procedure}

As shown in Figure 2, $50 \mathrm{~mL}$ spiked aqueous sample was added into $50.0 \mathrm{~mL}$ volumetric flask and after immersing in 
Table 1. Selected ions used for the quantification and qualification of phthalate esters by GC-MS (SIM mode)

\begin{tabular}{lcccc}
\hline Analyte & Abbreviation & Retention time / min & Confirmation ions $(\mathrm{m} / z)$ & Quantification ion $(\mathrm{m} / z)$ \\
\hline Di methyl phthalate & DMP & 13.4 & $77,135,163,194$ & 163 \\
Di ethyl phthalate & DEP & 15.8 & $121,149,177,222$ & 149 \\
Di butyl phthalate & DBP & 21.3 & $121,149,205,223$ & 149 \\
Bis(2-ethylhexyl) phthalate & BEHP & 26.3 & $45,72,121,149$ & 149 \\
Butyl benzyl phthalate & BBP & 28.5 & $91,149,206,238$ & 149 \\
Di $n$-octyl-phthalate & DNOP & 30.4 & $149,179,261,79$ & 149 \\
\hline
\end{tabular}

an ultrasonic bath, 150 to $500 \mu \mathrm{L}$ of toluene was injected. Then an ultrasonic bath (frequency $40 \mathrm{~Hz}$, power $80 \mathrm{~W}$ ) was turn on for $3 \mathrm{~min}$. The cloudy mixture was formed and transferred into a homemade long glass tube and subjected to aeration process using an air pump with flow rate of $2 \mathrm{~mL} \mathrm{~min}^{-1}$ until phase separation occurred and the aqueous phase became clear. Afterwards, the extraction phase was moved to the narrow neck of the tube by the injection of water. Then, the extraction phase on the top of the solution was collected using a micro syringe. Finally, $1.0 \mu \mathrm{L}$ of the collected organic solvent was injected into the GC-MS.

\section{Results and Discussion}

There are different factors that can affect the microextraction process, such as: selection of suitable extraction solvent and its volume, $\mathrm{pH}$, ionic strength, sonication and flotation time, and sample volume. It is very important to optimize them in order to obtain good recovery, high enrichment factor (EF) and low limit of detection (LOD).

The equations that describe the effects of several parameters on the efficiency of the proposed
IT-UAA-LLME method are similar to those of LLE and are given as follows:

$$
\begin{aligned}
& E_{\mathrm{r}}=\frac{n_{\mathrm{ex}}}{n_{\mathrm{ini}}}=\frac{C_{\mathrm{f}}^{\mathrm{ex}} V_{\mathrm{ex}}}{C_{\mathrm{ini}}^{\mathrm{sam}} V_{\mathrm{sam}}} \\
& P_{\mathrm{f}}=\frac{C_{\mathrm{f}}^{\mathrm{ex}}}{C_{\text {ini }}^{\mathrm{sam}}}
\end{aligned}
$$

where $P_{\mathrm{f}}$ is the preconcentration factor, $E_{\mathrm{r}}$ is the extraction recovery, $C_{\mathrm{f}}^{\text {ex }}$ and $C_{\text {ini }}^{\text {sam }}$ are the final concentration in the extracting phase, and initial analyte concentrations in the source phase, respectively. $n_{\mathrm{ex}}$ is the number of moles of the analyte extracted into the extracting phase, and $n_{\text {ini }}$ is the total number of moles of the analyte; $V_{\mathrm{ex}}$ and $V_{\mathrm{sam}}$ are the final (separated) volume of extracting phase and volume of sample phase.

\section{Effect of solvent and volume of solvent}

Selection of an appropriate solvent is an important parameter in extraction methods. When choosing a solvent, the solvent characteristics including the distribution

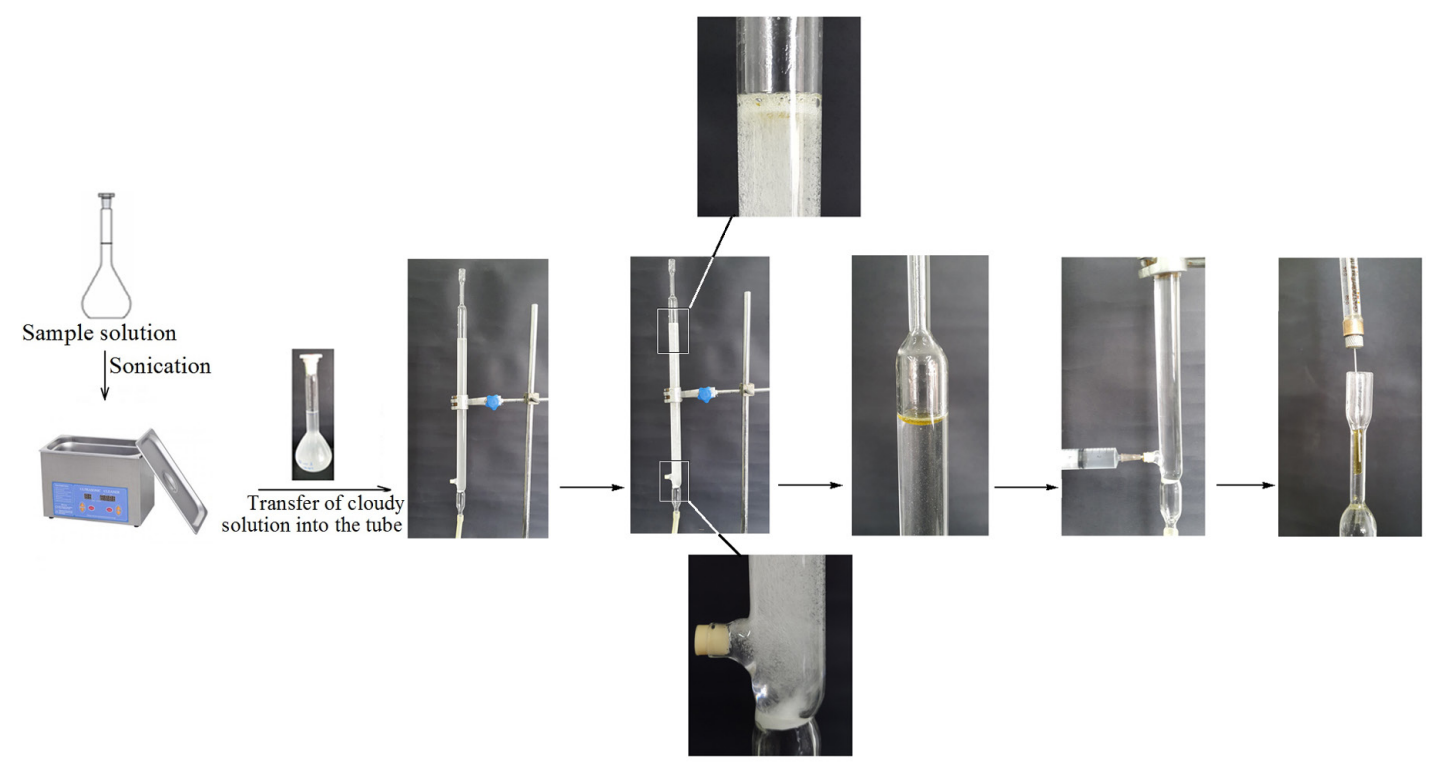

Figure 2. Photography of different steps in IT-UAA-LLME. 
coefficient of the target analytes, density, water solubility, volatility, disperse ability, surface tension, viscosity and compatibility of solvent with the analytical instrument, are important factors to be considered. Water-immiscible solvents, such as toluene, butyl acetate and octane, were examined for the extraction of PEs from water samples. Toluene has more compatibility with GC-MS, less water solubility than butyl acetate, and has good extraction efficiency in comparison with other solvents (Figure 3).

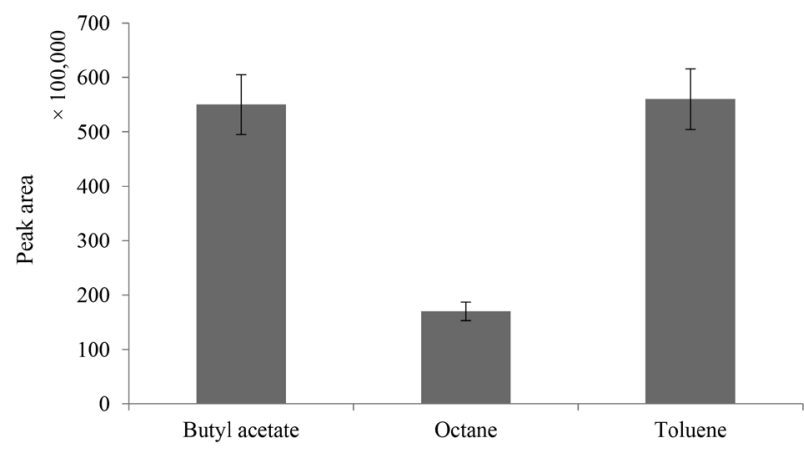

Figure 3. Effect of solvent type on overall (sum of area for six PEs) extraction of PEs from spiked water sample. Conditions: volume of sample: $50 \mathrm{~mL}$, concentration of each PEs: $10 \mathrm{ng} \mathrm{mL}^{-1}, \mathrm{pH}: 7$, sonication time: $3 \mathrm{~min}$, aeration.

In order to evaluate the effect of volume of toluene, different volumes of toluene $(150,200,300,400$, and $500 \mu \mathrm{L}$ ) were examined using the same extraction procedure. The obtained results are shown in Figure 4. The results showed that the peak area (concentration) of the extracted PEs decreased with increasing volume of toluene in the studied range. The PEs were diluted with increasing volume of toluene, and thus $150 \mu \mathrm{L}$ of solvent was chosen as the optimized volume for analysis.

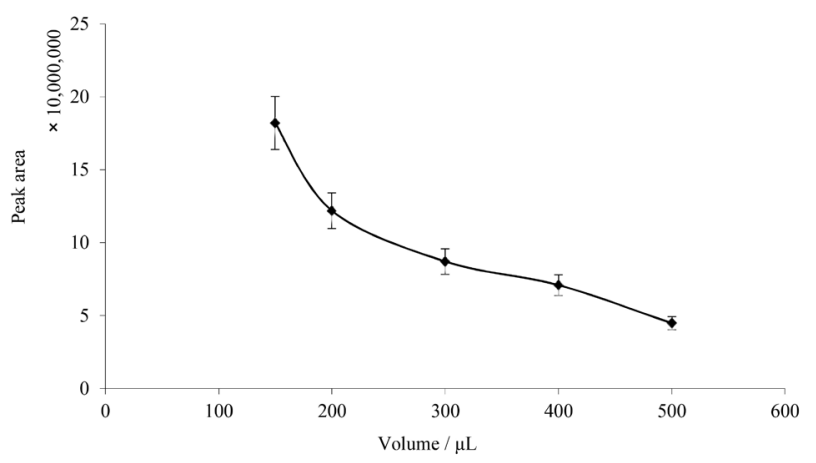

Figure 4. Effect of volume of toluene on overall (sum of area for six PEs) extraction of PEs from spiked water sample. Conditions: volume of sample: $50 \mathrm{~mL}$, concentration of each PEs: $10 \mathrm{ng} \mathrm{mL}^{-1}, \mathrm{pH}: 7$, sonication time: 3 min, flotation time: $10 \mathrm{~min}$.

\section{Effect of sonication and aeration time}

Sonication is an important stage, which makes the organic extraction solvent to become well dispersed into the aqueous solution and results in an excellent enrichment. An ultrasound-assisted process can promote the surface of the extraction solvent and accelerate the formation of a cloudy solution. In this method, toluene was quickly disrupted by sonication and dispersed in the water sample as fine droplets. Hence, the ultrasonic time plays an important role in this procedure. However, there are disadvantages in prolonging the ultrasonication time, such as loss of extraction solvent and analytes. The effect of ultrasonication time was evaluated in the range of 1-6 min. As shown in Figure 5, sonication for 3 min was enough to form a complete cloudy solution. Hence, $3 \mathrm{~min}$ was chosen for the dispersive procedure. However, organic solvents tend to volatilize under ultrasonic radiation and it is possible that long sonication time increased the temperature of ultrasonic bath, resulting in loss of these analytes.

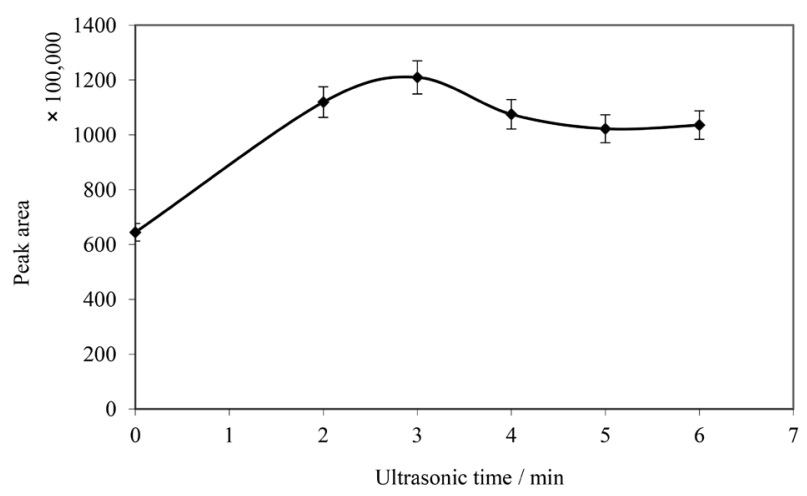

Figure 5. Effect of ultrasonic time on overall (sum of area for six PEs) extraction of PEs from spiked water sample. Conditions: volume of sample: $50 \mathrm{~mL}$, concentration of each PEs: $10 \mathrm{ng} \mathrm{mL}^{-1}$, volume of toluene: $150 \mu \mathrm{L}$, flotation time: $10 \mathrm{~min}$.

In the proposed method, phase separation was performed using aeration process instead of conventional centrifugation step. The aeration process caused phase separation and increased the extraction efficiency. To increase the extraction efficiency, the number and size of bubbles are important. Therefore, a sintered glass (No. 0) was mounted at the end of the glass tube. Furthermore, air pressure was maintained in a way that the solution did not overflow and aeration continued until the sample solution became clear. As shown in Figure 6, with increase in aeration time, the extraction efficiency of analytes increased up to $10 \mathrm{~min}$ and then leveled off. Therefore, 15 min was selected as the optimum aeration time for further experiments.

Type and amount of salt

Ionic strength is regarded as one of the parameters 


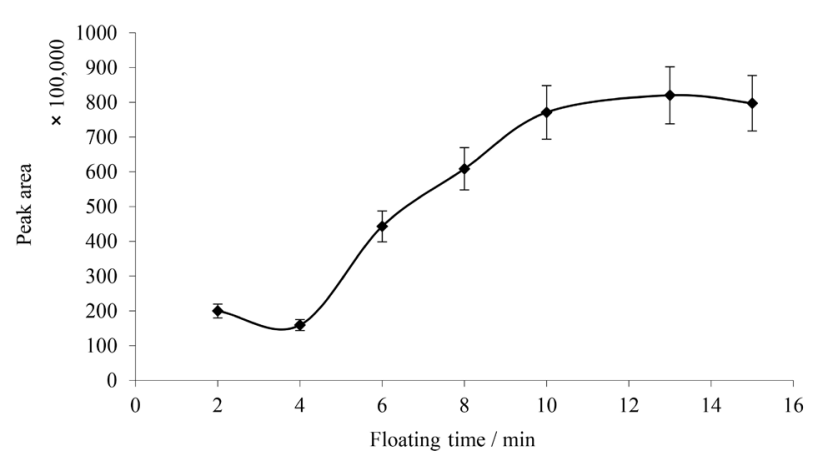

Figure 6. Effect of aeration time on overall (sum of area for six PEs) extraction of PEs from spiked water sample. Conditions: volume of sample: $50 \mathrm{~mL}$, concentration of each PEs: $10 \mathrm{ng} \mathrm{mL}^{-1}, \mathrm{pH}: 7$, volume of toluene: $150 \mu \mathrm{L}$, ultrasonic time: $3 \mathrm{~min}$.

affecting extraction efficiency. In this study, various salts, including sodium chloride, magnesium sulfate and magnesium chloride, were used at equal concentration. Extraction efficiency with magnesium sulfate was two orders of magnitude higher than other salts. Therefore, the effect of increasing the ionic strength of the sample was evaluated by adding $\mathrm{MgSO}_{4}(0.1-2 \% \mathrm{~m} / \mathrm{m})$ into the sample solution. The peak area of PEs was increased by increasing the amount of salt up to $0.3 \% \mathrm{~m} / \mathrm{m}$ and then decreased (data is not given).

\section{Analytical results}

The performance of this method was investigated under optimized conditions. A series of standard solution containing 5, 50, 100, 200, 500, 1000, 2000, $4000 \mathrm{ng} \mathrm{mL}^{-1}$ of PEs were used for plotting the calibration curve. The calibration curves were constructed by plotting the peak areas versus the concentrations of the individual analyte with triplicate measurements. The LODs, based on a signal-to-noise ratio $(\mathrm{S} / \mathrm{N})$ of 3 and the limits of quantification (LOQs), based on an $\mathrm{S} / \mathrm{N}$ of 10 , were calculated. The LODs were obtained in the range of $0.1 \mathrm{ng} \mathrm{mL} \mathrm{m}^{-1}$ (for BEHP) to $2.11 \mathrm{ng} \mathrm{mL}^{-1}$ (for di $n$-octylphthalate, DNOP) and the enrichment factors ranged from 100 to 330. The results are shown in Table 2.

\section{Application of the proposed method to real sample}

A series of polyethylene terephthalate bottle including three widely consumed drinking water and one cola were purchased from various local supermarkets in Tehran, Iran, for real sample analysis to evaluate the performance of the proposed method under optimized conditions. Figures 7, 8 and 9 show the typical chromatograms of standard solution and water sample spiked with the target analytes. The recoveries for the target analytes in the spiked real samples are listed as relative recoveries in Table 3 .

To assess the precision of the measurement, repeatability study was carried out by performing three individual experiments at a concentration of $10 \mathrm{ng} \mathrm{mL} \mathrm{mL}^{-1}$ for each of the PEs. The repeatability of the IT-UAA-LLME method was evaluated via analysis of three replicate experiments by spiking the deionized water with three analytes at known concentrations. To study the intraday and inter-day precision, spiked samples were extracted using the optimized proposed method in one and three different days in triplicate. The results are shown in Table 3. The relative standard deviations (RSDs) varied from 2 to $8 \%$.

The presence of two PEs (BBP and DNOP) was verified in mineral water and cola by comparing the mass data of 149 scans (retention times 28.5 and 30.4 min, respectively) with those of the studied PEs. It is shown that the migration of PEs from the vessel to the solution increased in acidic $\mathrm{pH}$.

\section{Comparison of the proposed method with other methods}

The determination of phthalate esters in water samples using the proposed method was compared with other methods and the results are shown in Table 4. The results demonstrated that the proposed method has both good LODs and linear range and better repeatability (RSDs) than the other methods. Furthermore, this method has higher EFs and easier operation steps in comparison with other methods in screening phthalate esters in

Table 2. Analytical features of IT-UAA-LLME method

\begin{tabular}{|c|c|c|c|c|}
\hline PEs & $\mathrm{DLR} /\left(\mathrm{ng} \mathrm{mL}^{-1}\right)$ & $\mathrm{R}^{2}$ & $\mathrm{LOD} /\left(\mathrm{ng} \mathrm{mL} \mathrm{m}^{-1}\right)$ & $\mathrm{LOQ} /\left(\mathrm{ng} \mathrm{mL}^{-1}\right)$ \\
\hline DMP & 6-1000 & 0.993 & 1.68 & 5.56 \\
\hline DEP & $5-1000$ & 0.995 & 0.86 & 2.84 \\
\hline DBP & $5-1000$ & 0.990 & 0.75 & 2.46 \\
\hline BEHP & $5-1000$ & 0.991 & 0.10 & 0.34 \\
\hline BBP & $6-1000$ & 0.992 & 1.67 & 5.50 \\
\hline DNOP & $7-1000$ & 0.996 & 2.11 & 6.97 \\
\hline
\end{tabular}

DLR: Dynamic linear range; LOD: limit of detection; LOQ: limit of quantification. 


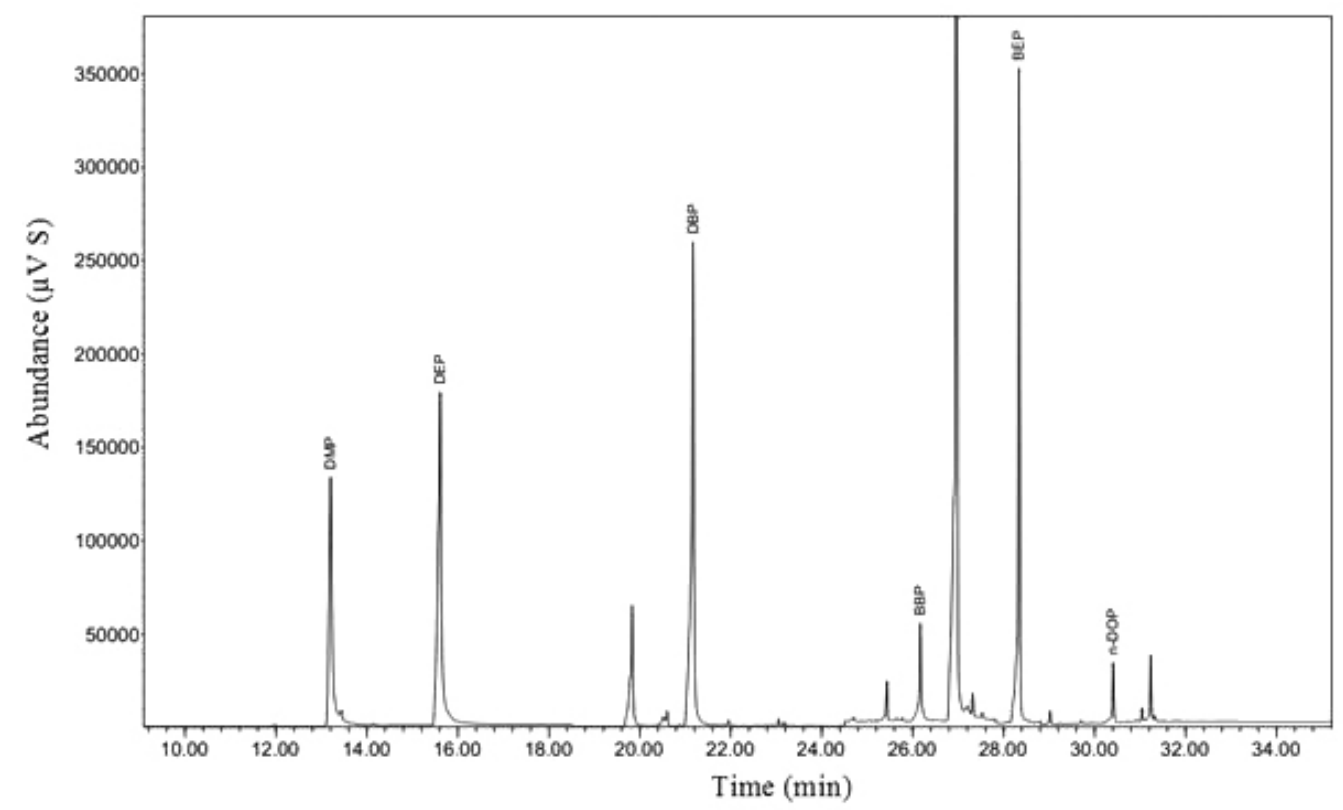

Figure 7. GC-MS chromatogram of standard solution.

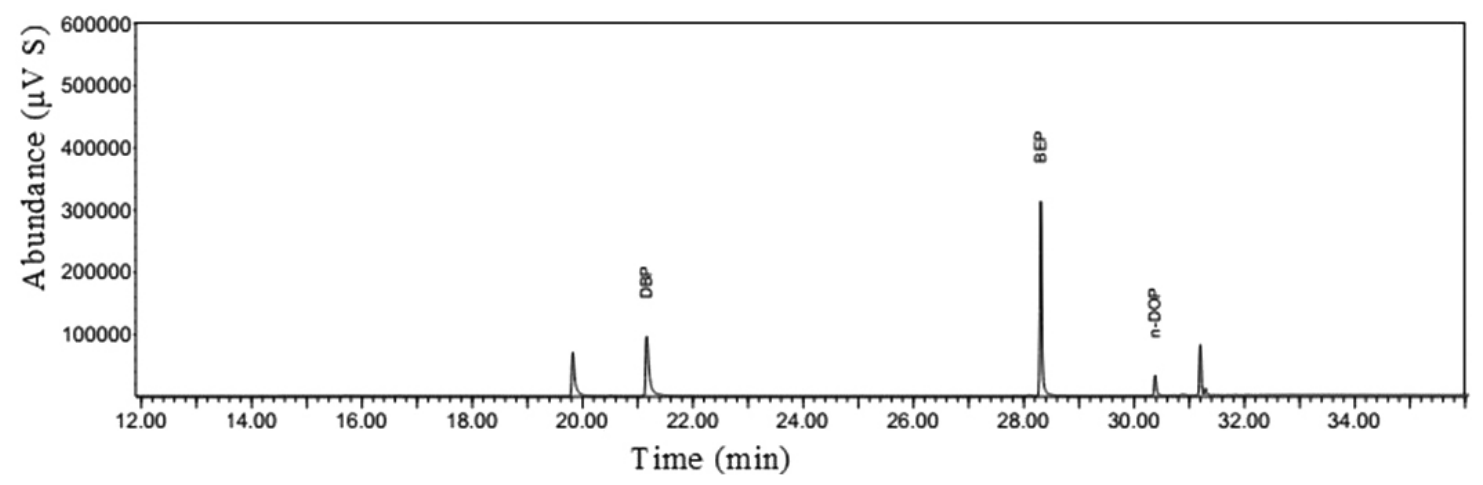

Figure 8. GC-MS chromatogram of real sample.

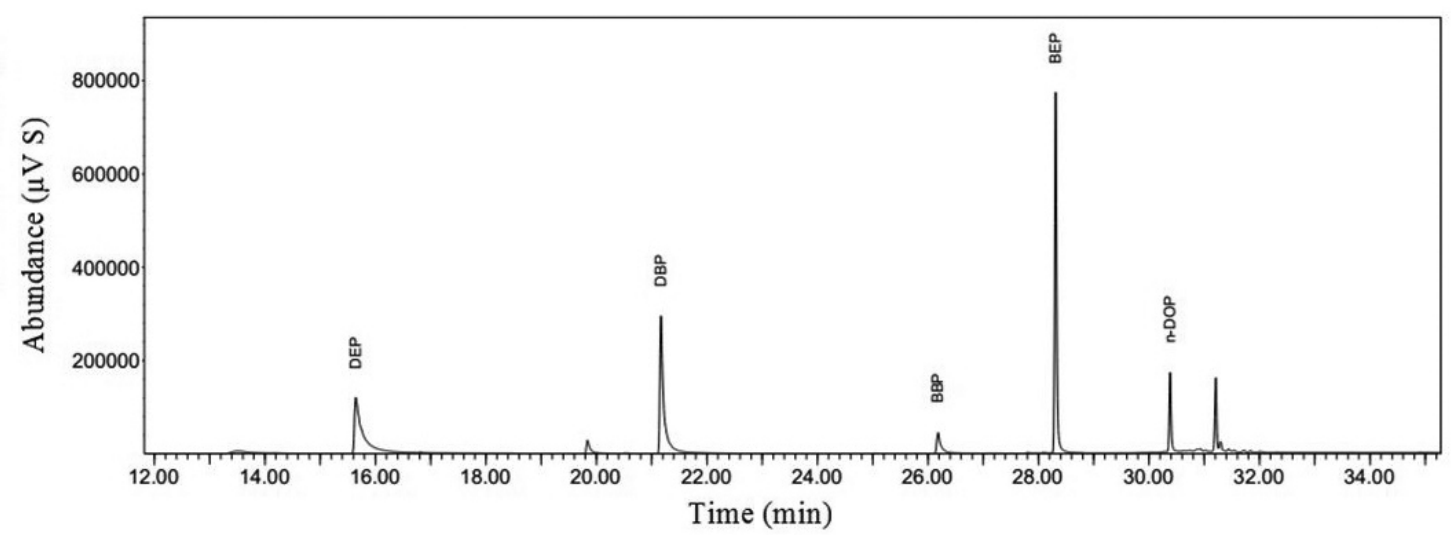

Figure 9. GC-MS chromatogram of spiked sample.

aqueous samples. We don't use chlorinated solvent and thus our method is greener than other dispersive liquidliquid microextraction, but some of the published article provided better analytical features than the work. Each work has advantages and disadvantages and we coupled ultrasonic dispersion and air assisted liquid-liquid microextraction. Thus our method is a high throughput sample preparation technique. 
Table 3. Accuracy data for PEs spiked in real samples

\begin{tabular}{|c|c|c|c|c|c|c|c|c|c|}
\hline \multirow[b]{2}{*}{ Sample } & \multirow{2}{*}{$\begin{array}{l}\text { Spiked / } \\
\left(\mathrm{ng} \mathrm{mL}^{-1}\right)\end{array}$} & \multicolumn{2}{|c|}{ Mineral water 1} & \multicolumn{2}{|c|}{ Mineral water 2} & \multicolumn{2}{|c|}{ Mineral water 3} & \multicolumn{2}{|c|}{ Cola } \\
\hline & & $\begin{array}{c}\text { Found / } \\
\left(\mathrm{ng} \mathrm{mL}^{-1}\right)\end{array}$ & $\begin{array}{c}\text { Recovery } \pm \\
\text { RSD / \% }\end{array}$ & $\begin{array}{c}\text { Found / } \\
\left(\mathrm{ng} \mathrm{mL}^{-1}\right)\end{array}$ & $\begin{array}{c}\text { Recovery } \pm \\
\text { RSD / \% }\end{array}$ & $\begin{array}{c}\text { Found / } \\
\left(\mathrm{ng} \mathrm{mL}^{-1}\right)\end{array}$ & $\begin{array}{c}\text { Recovery } \pm \\
\text { RSD / \% }\end{array}$ & $\begin{array}{c}\text { Found / } \\
\left(\mathrm{ng} \mathrm{mL}^{-1}\right)\end{array}$ & $\begin{array}{c}\text { Recovery } \pm \\
\text { RSD / \% }\end{array}$ \\
\hline \multirow[t]{2}{*}{ DMP } & - & N.D. & - & N.D. & - & N.D. & - & N.D. & - \\
\hline & 10.0 & 10.3 & $103 \pm 4$ & 9.6 & $96 \pm 3$ & 8.9 & $89 \pm 2$ & 11.2 & $112 \pm 6$ \\
\hline \multirow[t]{2}{*}{ DEP } & - & N.D. & - & N.D. & - & N.D. & - & 0.7 & - \\
\hline & 10.0 & 10.8 & $108 \pm 7$ & 9.4 & $94 \pm 7$ & 10.7 & $107 \pm 3$ & 9.7 & $90 \pm 5$ \\
\hline \multirow[t]{2}{*}{ DBP } & - & 1.7 & - & 1.1 & - & 1.1 & - & 1.1 & - \\
\hline & 10.0 & 9.4 & $94 \pm 6$ & 8.7 & $87 \pm 6$ & 11.9 & $101 \pm 3$ & 10.3 & $92 \pm 5$ \\
\hline \multirow[t]{2}{*}{ BEHP } & - & N.D. & - & N.D. & - & 1.8 & - & N.D. & - \\
\hline & 10.0 & 11.1 & $111 \pm 5$ & 9.3 & $93 \pm 5$ & 11.6 & $98 \pm 5$ & 8.8 & $88 \pm 4$ \\
\hline \multirow[t]{2}{*}{ BBP } & - & 4.1 & - & 2.9 & - & 5.5 & - & 12.7 & - \\
\hline & 10.0 & 14.9 & $108 \pm 6$ & 12.3 & $94 \pm 5$ & 16.5 & $110 \pm 5$ & 23.7 & $110 \pm 5$ \\
\hline \multirow[t]{2}{*}{ DNOP } & - & 2.0 & - & 2.6 & - & 3.4 & - & 10.6 & - \\
\hline & 10.0 & 12.3 & $103 \pm 8$ & 12.4 & $98 \pm 3$ & 12.8 & $94 \pm 4$ & 19.7 & $91 \pm 5$ \\
\hline
\end{tabular}

N.D.: not detected.

Table 4. Comparison of IT-UAA-LLME with other methods for the determination of PEs in liquid samples

\begin{tabular}{|c|c|c|c|c|c|c|}
\hline Method & $\begin{array}{l}\text { Extraction } \\
\text { time / min }\end{array}$ & Extraction solvent & $\begin{array}{l}\text { Linear range / } \\
\left(\mathrm{ng} \mathrm{mL} \mathrm{mL}^{-1}\right)\end{array}$ & $\begin{array}{c}\mathrm{LOD} / \\
\left(\mathrm{ng} \mathrm{mL} L^{-1}\right)\end{array}$ & $\mathrm{RSD} / \%$ & Reference \\
\hline DLLME-HPLC $^{a}$ & 5 & carbon tetrachloride & $5-5000$ & $0.88-1.8$ & $4-6$ & 16 \\
\hline SPME-GC-MS & 90 & - & $0.02-10$ & $0.02-10$ & $4-6$ & 18 \\
\hline HF-LPME-GC-MS ${ }^{c}$ & 20 & toluene & $0.5-10$ & $0.02-10$ & $4-19$ & 19 \\
\hline IL-DLLME $^{\mathrm{d}}$ & 5 & {$\left[\mathrm{C}_{8} \mathrm{MIM}\right]\left[\mathrm{PF}_{6}\right]$} & $0.1-100$ & $0.1-100$ & $2-3.8$ & 20 \\
\hline LLPME-GC-MS $^{e}$ & 25 & 1-dodecanol & $0.05-100$ & $0.2-0.5$ & $5-8$ & 21 \\
\hline $\operatorname{LLE}^{\mathrm{f}}$ & 30 & hexane & - & $1-6$ & $3-9$ & 22 \\
\hline $\mathrm{SPE}^{\mathrm{g}}$ & 30 & methylene chloride & - & $0.02-0.6$ & $1.5-14$ & 23 \\
\hline AALLME-GC-FID ${ }^{\mathrm{h}}$ & 5 & 1,1,2,2-tetrachloroethane & $1-10000$ & $0.12-1.15$ & $2-3$ & 17 \\
\hline IT-UAA-LLME-GC-MS ${ }^{\mathrm{i}}$ & 10 & toluene & $5-1000$ & $0.1-2$ & $3-5$ & this work \\
\hline
\end{tabular}

${ }^{a}$ Dispersive liquid-liquid microextraction-high performance liquid chromatography; ${ }^{\mathrm{b}}$ solid phase microextraction-gas chromatography-mass spectrometry; chollow fiber liquid phase microextraction gas chromatography-mass spectrometry; dionic liquid dispersive liquid-liquid microextraction; ${ }^{\text {eliquid-liquid }}$ phase microextraction gas chromatography-mass spectrometry; fliquid-liquid extraction; ${ }^{\mathrm{g}}$ solid phase extraction; ${ }^{\mathrm{h}}$ air-assisted liquid-liquid microextraction gas chromatography flame ionization detector; in tube ultrasonic and air assisted liquid-liquid microextraction.

\section{Conclusions}

This study introduced a new, simple, cheap, sensitive ultrasonic and air assisted liquid-liquid microextraction, and employed a newly home-designed extraction tube device (IT-UAA-LLME) combined with GC-MS measurements. The proposed method demonstrated several advantages, such as simplicity, low toxicity to the environment and inexpensiveness and rapid utilization of phthalate esters in aqueous sample with low density extraction solvent. This method has several advantages over previous methods in this context: using a suitable sintered glass at the bottom of glass tube results in producing tiny air bubble and prevents sample solution from overflowing, proper dispersion of extraction solvent by using ultrasonic waves, and good reproducibility due to reproducible production of tiny air bubble and fine droplet of extraction solvent. To assess the application of this method, it was successfully applied to determine phthalate esters in several waters.

\section{Acknowledgment}

The authors of this survey would like to thank the Danesh Pajohan Payesh Amin Company.

\section{References}

1. Aparicio, I.; Santos, J. L; Alonso, E.; J. Anal. Chim. Acta 2007, $584,455$.

2. Rios, J.; Morales, A.; Marquez-Ruiz, Z.; Talanta 2010, 80, 2076. 
3. Wang, F.; Yao, J.; Sun, K.; Xing, Z.; Environ. Sci. Technol. 2010, 44, 6985.

4. Xueju, L. V.; Hao, Y.; Jia, Q.; J. Chromatogr. Sci. 2013, 51, 632.

5. Zhang, H.; Chen, X.; Jiang, X.; J. Anal. Chim. Acta 2011, 689, 137.

6. Liang, P.; Xu, J.; Li, Q.; J. Anal. Chim. Acta 2008, 609, 53.

7. Wu, Q.; Liu, M.; Ma, X.; Wang, W.; Wang, Ch.; Zang, X.; Wang, Zh.; J. Microchim. Acta 2012, 177, 23.

8. Luo, Y. B.; Yu, Q. W.; Yuan, B. F.; Feng, Y. Q.; Talanta 2012, 90, 123.

9. Ezoddin, M.; Abdi, K.; Esmaeili, N.; Microchem. J. 2016, DOI: 10.1016/j.microc.2016.07.005.

10. Ezoddin, M.; Abdi, K.; Lamei, N.; Talanta 2016, 153, 247.

11. Sricharoen, P.; Limchoowong, N.; Techawongstien, S.; Chanthai, S.; Food Chem. 2016, 203, 386.

12. Farajzadeh, M. A.; Djozan, D.; Afshar Mogaddam, M. R.; Norouzi, J.; J. Sep. Sci. 2012, 35, 742.

13. Leng, G.; Chen, W.; Zhang, M.; Huang, F.; Cao, Q.; J. Sep. Sci. 2014, 37, 684.

14. Liao, Q. G.; Li, W. H.; Lou, L. G.; J. Anal. Bioanal. Chem. 2013, 405, 6791.

14. Abdollahzadeh, Y.; Yamini, Y.; Jabbari, A.; Esrafili, A.; Rezaee, M.; Anal. Methods 2012, 4, 830.
16. Liang, P.; Xu, J.; Li, Q.; J. Anal. Chim. Acta 2008, 609, 53.

17. Farajzadeh, M. R.; Afshar Mogaddam, M. R.; Anal. Chim. Acta 2012, 728, 31.

18. Penalver, A.; Pocurull, E.; Borrull, F.; Marce, R. M.; J. Chromatogr. A 2000, 872, 191.

19. Psillakis, E.; Kalogerakis, N.; J. Chromatogr. A 2003, 999, 145.

20. Zhang, H.; Chen, X.; Jiang, X.; J. Anal. Chim. Acta 2011, 689, 137.

21. Farahani, H.; Ganjali, M. R.; Dinarvand, R.; Norouzi, P.; Talanta 2008, 76, 718.

22. Kawahara, K.; Hodgeson, J. W.; EPA Method 506; Determination of Phthalate and Adipate Esters in Drinking Water by Liquid-Liquid Extraction or Liquid-Solid Extraction and Gas Chromatography with Photoionization Detection, U.S. Environmental Protection Agency: Cincinnati, Ohio, USA, 1995.

23. EPA Method 8061 A; Phthalate Esters by Gas Chromatography with Electron Capture Detection (GC/ECD), U.S. Environmental Protection Agency: Cincinnati, Ohio, USA, 1996.

Submitted: May 29, 2016

Published online: August 31, 2016 